\title{
ARE THERE GIANT VORTICES NEAR SOLAR CIRCLE?
}

\author{
A.M. FRIDMAN, O.V. KHORUZHII, V.V. LYAKHOVICH AND \\ V.S. AVEDISOVA \\ Institute of Astronomy, Russian Academy of Sciences \\ 48 Pyatnitskaya St., Moscow, 109017, Russia
}

\begin{abstract}
The analysis of the observational line-of-sight radial velocity field of molecular clouds, connecting with young stars, has strengthened the Fridman's hypothesis (1994) on the possible existence of anticyclone in the solar neighborhood. Anticyclones are located near corotation radius of the observed spiral arms, a number of which is equal to a number of vortices. Our calculations show that the four-vortices model fits observational data fairly well.
\end{abstract}

We shall not use any theoretical conception on the nature of spiral arms generation (bar, selfgravitational or hydrodynamical mechanisms, etc.). We shall base on the treatment of the observational data.

\section{Data}

The observational velocity field in the visible solar neighborhood was constructed on the basis of the photometric distances to the hot stars, exciting the diffuse nebula, and the radial velocities of the molecular clouds, connecting with these nebula. For a distance determination, $r$, we used all available new data, concerning the photometry and spectral classification of the stars. In particular new measurements of UBV and spectral classification of LS catalogue stars were used, and also associations of HII regions with open clusters for more than 90 star forming regions. Absolute magnitudes have been derived with common calibration curve (Landolt-Bornstein, 1982). Almost all BBW nebula with distances determined by Brand and Blitz (1993) were included in the sample. The radial velocity, $V_{l s r}$, have been taken from the Catalogue of observational data in the galactic star-forming regions (Avedisova 1994). Fig. 1 shows the radial line-of-sight velocity field of 316 objects. 


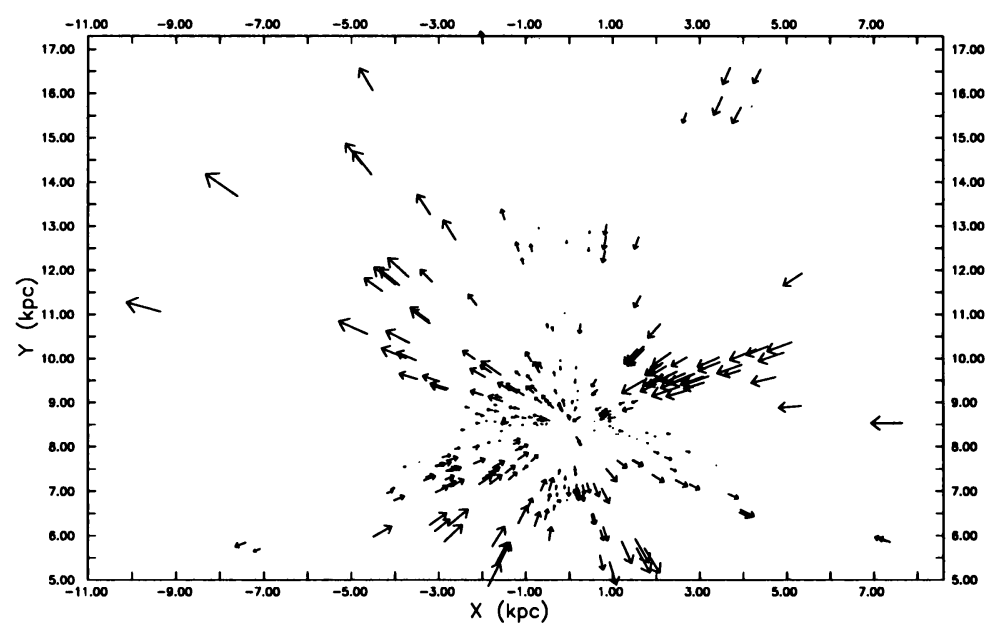

Figure 1. Line-of-sight radial velocity field of the sample of 316 objects. The Sun is at $(0,8.5)$

\section{Model of pure circular orbits}

Assuming pure circular orbits for the gas and stars, the circular rotation velocity, $\Theta_{c}$, of any object with known galactocentric distance $R$ and radial velocity $V_{l s r}$ (Brand and Blitz 1993) is equal to

$$
\Theta_{c}=\frac{R V_{l s r}}{R_{0} \sin l \cos b}+\frac{\Theta_{0} R}{R_{0}}
$$

where $R_{0}$ and $\Theta_{0}$ are the galactocentric distance of the Sun and the circular rotation velocity at the position of the Sun; $l$ and $b$ are the galactic longitude and latitude. Below we take $R_{0}=8.5 \mathrm{kpc}$., $\Theta_{0}=220 \mathrm{~km} / \mathrm{s}$. Substituting our data in the right hand of (1) we find $\Theta_{c}$ as triangles in Figs.2-3.

\section{Systematic deviations from the pure circular motion}

If to overlap the rotation curve of the Herthern Here (Fig. 2a) on the Southern one (Fig. 2b) we can see some discrepancy between them. Fig. 3a shows that in the azimuth range $5^{\circ}<\theta<15^{\circ}$ cloud motions deviate from a circular rotation the most strongly. Fig. $3 \mathrm{~b}$ shows that in the galactocentric region $9 \mathrm{kpc}<R<11 \mathrm{kpc}$ the cloud motion deviate from the circular rotation strongly. From Figs. 2-3 it follows that the strongest influence on the cloud motions is exerted by the Perseus arm. That is well known result (Burton 1966; Shane \& Bieger-Schmith 1966; Rickard 1968; Kerr 1969; Humphereys 1970,1976; Brand and Blitz 1993). 

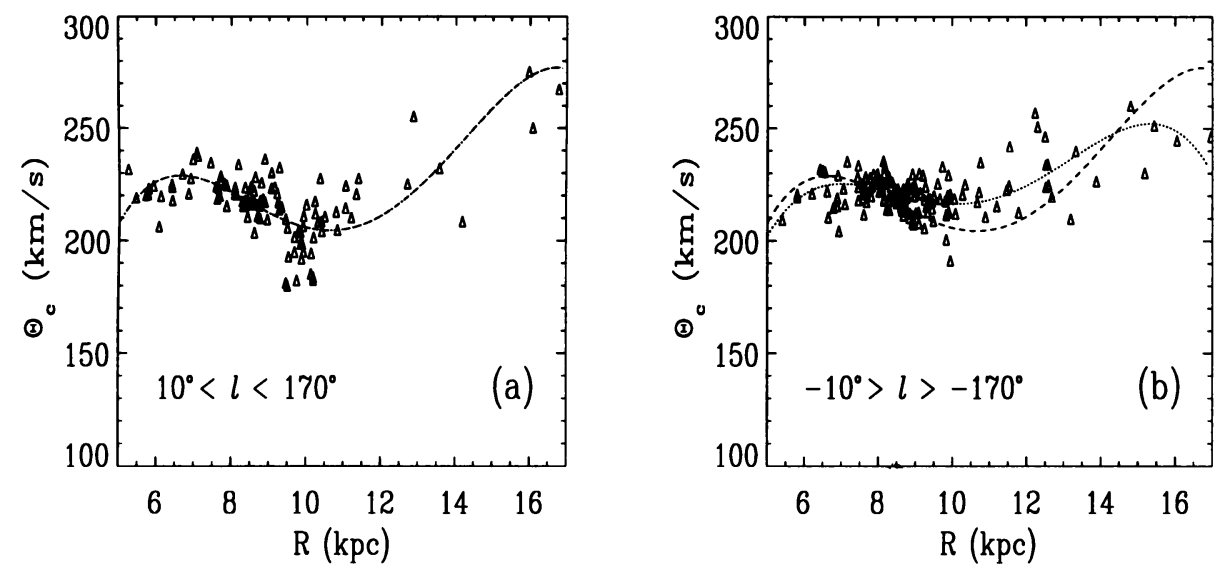

Figure 2. The circular rotation velocity $\Theta_{c}$ as a function of the galactocentric distance $R$ for objects of the Northern (a) end Southern (b) Hemispheres. There are differences between the Northern (broken line) and Southern (dotted line) rotation curves
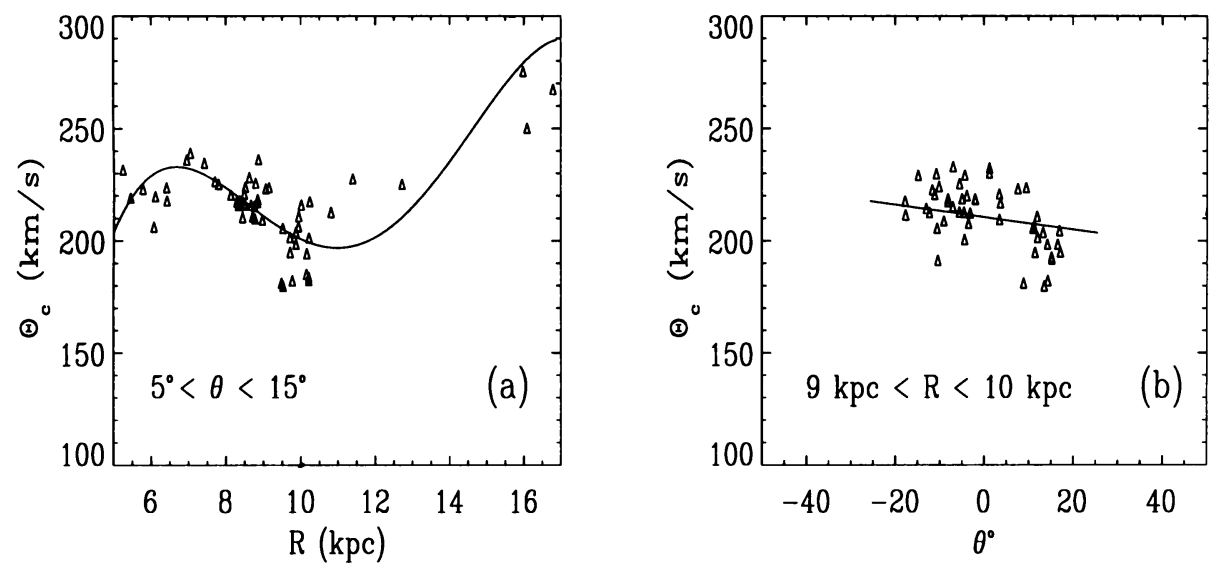

Figure 3. The circular rotation velocity $\Theta_{c}$ as a function of (a) the galactocentric distance $R$ for the range of galactocentric azimuth $5^{\circ}<\theta<15^{\circ}$, (b) the azimuth $\theta$ for the range of galactocentric distances $9 \mathrm{kpc}<R<10 \mathrm{kpc}$ 
The average velocity in the direction of the galactic center $\left(-10^{\circ}<l<\right.$ $\left.10^{\circ}\right)$ in the region of the Sagittarius-Carina arm is equal to $\Pi^{\prime} \equiv \Pi-\Pi_{0}=$ $(-7.4 \pm 1.3) \mathrm{km} / \mathrm{s}$, where $\Pi$ and $\Pi_{0}$ are the radial galactocentric velocity of objects and LSR, respectively. The same value according to Avedisova $(1986)$ is $\Pi^{\prime}=(-6.9 \pm 2.2) \mathrm{km} / \mathrm{s}$. In the anticenter direction $\left(170^{\circ}<l<\right.$ $\left.190^{\circ}\right)$ in the region of the Perseus arm that is $\Pi^{\prime}=(-4.4 \pm 1.7) \mathrm{km} / \mathrm{s}$. The last value according to Velden (1970) is $\Pi^{\prime} \approx-3.3 \mathrm{~km} / \mathrm{s}$, Stark (1984) $\Pi^{\prime}=(-3.1 \pm 1.0) \mathrm{km} / \mathrm{s}$, Brand and Blitz $(1993) \Pi^{\prime}=(-3.8 \pm 1.8) \mathrm{km} / \mathrm{s}$.

And so we can see that the systematic deviations from the pure circular motion correlate with the location of the spiral arms. Therefore we try to explain all observed features on the base of the model which combines a circular rotation and motion in the spiral arms.

\section{The average motion of clouds consists of the circular rotation and motion in spiral arms}

The average (without a dispersion) motion of clouds is described by the following equation

$$
\frac{V_{l s r}}{\cos b}=\left(\frac{\Theta}{R}-\frac{\Theta_{0}}{R_{0}}\right) R_{0} \sin l-\frac{\Pi}{R}\left(R_{0} \cos l-d\right)+\Pi_{0} \cos l
$$

Formula (2) coincides with formula (14) of the Chapt.4 from Rohlfs (1977) (see Fig. 9), if we change $V_{l s r} / \cos b=v_{r}$ and $d / \cos b=r$.

Assume that the average velocity field is the superposition of the differential rotation of the Galaxy and motion in spiral waves (see Rohlfs 1977, Chapt.7 Eqs(12-13))

$$
\begin{aligned}
& \Theta=\Omega(R) R+A_{\Theta}(R) \cos \left(-m \Theta+F_{\Theta}(R)\right), \\
& \Pi=A_{\Pi}(R) \cos \left(-m \Theta+F_{\Pi}(R)\right)
\end{aligned}
$$

where $\Omega$ is the average angular velocity of the rotation, $A_{\Theta}, A_{\Pi}$ and $F_{\Theta}, F_{\Pi}$ are amplitudes and phases of the azimuthal and radial velocities in spiral waves, respectively, $m$ is number of spiral arms.

All parameters of our model were obtained by means of least-squares method (e.g. Eichhorn and Clary 1974, Jefferys 1981). In this paper we give results for $m=4$, which is consistent with four-arms structure of the Galaxy (see Blitz 1983, Malahova and Petrovskaya 1992).

\section{The radial motion of LSR and consequence of it}

In our model we obtained $\Pi_{0} \approx 5 \mathrm{~km} / \mathrm{s}$, that is consistent with that obtained by Fich et al., (1989), Brand and Blitz (1993), Fuchs, et al., (1994).

Taking into account the value $\Pi_{0}$ we can change from LSR frame to the galactocentric frame of reference. So we obtain, that the average velocity 


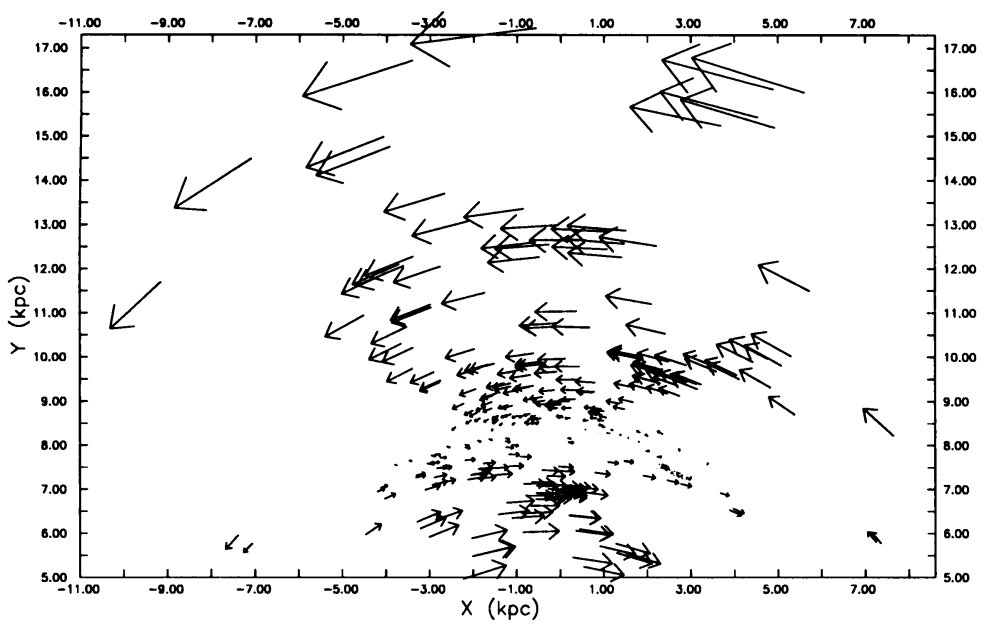

Figure 4. The full velocity field of the clouds has the form of a anticyclone with the center in the solar vicinity

$\Pi=\Pi^{\prime}+\Pi_{0}$ in the direction of the galactic center in the region of the Sagittarius-Carina arm is equal to $\Pi \approx-2 \mathrm{~km} / \mathrm{s}$, and in the direction of the anticenter in the region of the Perseus arm is equal to $\Pi \approx+1 \mathrm{~km} / \mathrm{s}$.

What does it mean that we obtained the value $\Pi$ with different signs for different arms?

It means that there is the corotation in the solar vicinity. Indeed, from a linear theory of spiral waves with a small pitch angle in WKB-approximation (e.g. Rohlfs 1977) we have $\Pi=-(\omega-m \Omega) \rho_{1} /\left(k \rho_{0}\right)$, where $\rho_{1}$ is the perturbation density. For trailing arms $k<0, \rho_{1}>0$ we have

$$
\Pi=\left\{\begin{array}{lll}
>0, & \Omega<\omega / m, & R>R_{c} \\
<0, & \Omega>\omega / m, & R<R_{c}
\end{array}\right.
$$

where $R_{c}$ is the corotation radius $\left(\omega-m \Omega\left(R_{c}\right)=0\right)$.

Let us show how the range of the corotation radius values depends on the range of the values $\Pi_{0}$ :

$$
\begin{array}{lll}
\text { if } \Pi_{0}<4 \mathrm{~km} / \mathrm{s} & \text { then } & R_{c}>11 \mathrm{kpc}, \\
\text { if } \Pi_{0}=4-7 \mathrm{~km} / \mathrm{s} & \text { then } & R_{c}=11-7 \mathrm{kpc}, \\
\text { if } \Pi_{0}>7 \mathrm{~km} / \mathrm{s} & \text { then } R_{c}<7 \mathrm{kpc} .
\end{array}
$$

Fig. 4 shows the velocity field model in the system which rotates with the angular velocity of the LSR. The full vector velocity field of clouds has the form of a anticyclone with the center near the solar vicinity and between spiral arms. This picture of the velocity field qualitatively looks like that was obtained by Fuchs et al., (1994) where data of proper motions 
(from PPM catalogue for objects by Brand and Blitz (1993)) were used. If the corotation radius is near the Solar distance then this anticyclone is stationary.

\section{Position of anticyclone centers with respect to spiral arms}

The positions of anticyclone centers with respect to spiral arms depends on the nature of the spiral generation (Lyakhovich et al., 1994). In the Table 1 we show positions of vortex centers in the observed case when $\kappa^{2} / 2 \Omega \equiv 2 \Omega(R)+R d \Omega(R) / d R>0$, where $\kappa$ is the epicyclic frequency. The first and second lines show the positions of vortex centers in the cases when a force of hydrodynamic pressure dominates a selfgravitation force and vice versa. The third line corresponds to the case when a gaseous disk is perturbed by some external perturbed force (e.g. a bar, stellar spirals, a satellite, etc.). So the location of vortex centers points to the generation mechanism of spiral arms.

TABLE 1. Centers of anticyclons

\begin{tabular}{cc}
\hline hydrodynamic & on spiral arms \\
\hline selfgravitation & between spiral arms \\
\hline $\begin{array}{c}\text { External perturbing } \\
\text { gravitational potential }\end{array}$ & $\begin{array}{c}\text { centers of vortices } \\
\text { have intermediate position }\end{array}$ \\
\hline
\end{tabular}

In our case the position of the vortex center shows that the selfgravitation force dominates the force of hydrodynamic pressure. The center of the anticyclone show a possibility of the existence of a bar structure or non-axisymmetric gravitational potential in the Milky Way.

\section{Conclusion}

1. Near the corotation region of the observed spiral structure, anticyclones must exist independently on the nature of the generation of spiral arms.

2. The number of anticyclones is equal to the number of spiral arms.

3. Azimuthal position of the anticyclone centers with respect to spiral arms depends on the nature of spiral generation.

4. If hydrodynamical force dominates, centers of anticyclones are located on the arms. If self-gravitating force dominates, centers of anticyclons are located between arms. Intermediate position of anticyclone centers is possible if the spiral structure is generated by external force (e.g. by a bar). 
5. If the radial galactocentric velocity of LSR is in the range of 4-7 km $\mathrm{s}^{-1}$, the corotation radius of the observed spiral structure lies near the solar circle.

6. The systematic deviations from the pure circular motion are observed in the solar vicinity. These deviations correlate with the location of the spiral structure.

7. The model velocity field combined the circular rotation and the motion in spiral arms describes all main peculiarities of velocity field observed in the solar vicinity.

8. In the model the anticyclone center lies near the solar vicinity.

\section{Acknowledgements}

We thank the Russian Fund of Fundamental Investigations (grant 93-0217248) for financial support.

\section{References}

Avedisova V. S. (1994) Catalogue of observational data in galactic star-forming regions, Version 1.2 (in preparation).

Avedisova V. S. (1986) Sov. Astron. Lett. 11, 6, 378.

Blitz, L. (1983) In "Surveys of the Southern Galaxy", eds. W.B.Burton and F.P.Israel, Dordrecht, 117.

Brand, J. and Blitz, L., (1993) Astron. Asrophys. 275, 67.

Burton, W.B. (1966) Bull. Astron. Inst. Neth. 18, 247.

Fridman, A.M. (1994) In "Physics of the Gaseous and Stellar Disks of the Galaxy", ed. I.R.King, Astron. Soc. of the Pacific, 66, 15.

Fich, M., Blitz, L., Stark, A.A., (1989) ApJ 342, 272.

Fuchs, B., Frink, S., Röser, S., Wielen, R. (1994) Proceedings of the IAU Symposium 169 " Unsolved Problems of the Milky Way" held in Den Haag, the Netherland, 25-28 August.

Humphereys, R.M. (1970) AJ 75, 602.

Humphereys, R.M. (1976) ApJ 206, 114.

Jefferys, W. H., Astron. J. (1981) 86, 149.

Kerr, F.J. (1969) ARA\&A 7, 39.

Landolt-Bornstein (1982) Numerical Data and Functional Relationships in Science an Technology, New Series, Group VI, 2, pp.15-18; 451; 453-455.

Lyakhovich, V.V. Fridman, A.M., Khoruzhii, O.V., (in preparation).

Malahova, Yu.N., Petrovskaya, I.V. (1992) Astron. and Astroph. Transactions, 1, 221.

Eichhorn, H.K. and Clary, W.G. (1974) Mon. Not. R. Astron. Soc. 166, 425.

Rohlfs, K. (1977) Lectures on density wave theory, Springer-Verlag, Berlin, Heidelberg, New York.

Rickard J.J. (1968) ApJ 152, 1019.

Shane, W.W., Bieger-Schmith, G.P. (1966) Astron. Inst. Neth. 18, 263.

Stark A.A. (1984) Ap. J. 281, 624.

Velden L. (1970) IAU Symp. 38, 164. 